\title{
Computational fluid dynamics analysis and PIV validation of a bionic vortex flow pulsatile LVAD
}

\author{
Liang Xu, Ming Yang*, Lin Ye and Zhaopeng Dong \\ Department of Instrument Science and Engineering, Shanghai Jiao Tong University, Shanghai, China
}

\begin{abstract}
.
BACKGROUND: Hemocompatibility is highly affected by the flow field in Left Ventricular Assistant Devices (LVAD).

OBJECTIVE: An asymmetric inflow and outflow channel arrangement with a $45^{\circ}$ intersection angle with respect to the blood chamber is proposed to approximate the vascular structure of the aorta and left atrium on the left ventricle. The structure is expected to develop uninterruptible vortex flow state which is similar to the flow state in human left ventricle.

METHODS: The Computational Fluid Dynamics (CFD) asymmetric model is simulated using ANSYS workbench. To validate the velocity field calculated by CFD, a Particle Image Velocimetry (PIV) experiment is conducted.

RESULTS: The CFD results show that the proposed blood chamber could generate a shifting vortex flow that would be redirected to the aorta during ejection to form a persistent recirculating flow state, which is similar to the echocardiographic flow state in left ventricle. Both the PIV and the CFD results show the development of a persistent vortex during the pulsatile period. Comparison of the qualitative flow pattern and quantitative probed velocity histories in a pulsatile period shows a good agreement between the CFD and PIV data.

CONCLUSION: The goal of developing persistent quasi intra-ventricle vortex flow state in LVAD is realized.
\end{abstract}

Keywords: Bionic vortex flow, left ventricular assist device, computational fluid dynamics, particle image velocimetry

\section{Introduction}

LVADs could promote favorable myocardial remodeling and prolong life in patients with heart failure [1-4]. Despite the recent trend towards continuous-flow LVADs, patients with pulsatile-flow LVADs have a higher chance of myocardial recovery, surplus hemodynamic energy, and demonstrate improved renal function, improved blood flow of the vital organs and a lower rate of gastrointestinal bleeding events [5-9]. However, pulsatile blood pumps suffer from design concerns which consist of poor reliability, decreased biocompatibility, inefficient hydraulic performance at small dimensions and the increased risk of thrombosis and hemolysis [10-12]. These challenges are mainly related to the flow states within the blood chamber which have been investigated extensively in the development of LVADs. For example, Eiji Okamoto et al. studied the effect of pump geometry on shear stresses and velocity distribution. They found a smaller space between inflow and outflow channel placed in parallel resulted in lower shear stresses and some local spiral flow around the diaphragm-housing junction which is in favor of reducing

\footnotetext{
${ }^{*}$ Corresponding author: Ming Yang, Department of Instrument Science and Engineering, Shanghai Jiao Tong University, Shanghai 200237, China. Tel./Fax: +86 0213420 4197; E-mail: myang@ sjtu.edu.cn.
} 

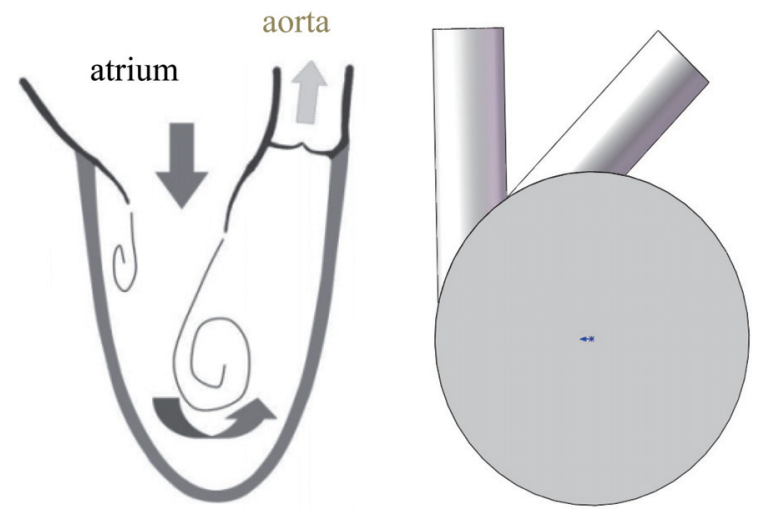

Fig. 1. Anatomical structure of left ventricle and the bionic blood chamber geometry.

hemolysis and enhancing pump washout [13]. By comparing valve orientations in the 50 cc Penn State device, Kreider et al. found that wall washing effect was more effective when the valve orientation was set at $45^{\circ}$ [14]. However, this vortex flow field was still confined to a 2-dimensional zone and lasted only for a short period of the filling phase. Using particle image velocimetry and computational fluid dynamics, Giridharan et al. investigated the design of a single port, valveless counter-pulsation device [15]. The device generated a 3-dimensional vortex flow during the entire filling phase, which showed low hemolysis and good wash effect to prevent thrombus deposition. However, washout was interrupted by the transition between filling and ejection phases. The swirling flow phenomenon was responsible for the quasi intraventricular flow state and good wash effect of LVADs. Ideally, the pulsatile LVADs should perform a complete vortex flow which was naturally optimized by human left ventricle to minimize the energy dissipation of the flow [16]. However, the vortex flow formed by current pulsatile blood pumps were either transient or interrupted by the transition between filling and ejection phase, which meant they could not form a complete circulation like the native ventricle.

In this study, we designed an asymmetric inflow and outflow channel arrangement with a $45^{\circ}$ intersection angle on blood chamber by imitating the cardiovascular structure on left ventricle trying to develop uninterruptible vortex flow state generated by human left ventricle. The model was analyzed in ANSYS workbench. The echocardiography was used to compare the flow state of CFD results in designed model and normal human left ventricle. And the computational results were validated by a PIV experiment.

\section{Methods}

\subsection{Blood chamber geometry and pump structure}

Figure 1 showed anatomical structure of left ventricle and the bionic structure of the model. The model was characterized by an asymmetric structure with a $45^{\circ}$ intersection angle between the inflow and outflow channels. The asymmetric structure in the blood chamber imitated the vascular structure of the human left ventricle. Two Medtronic Hall valves (Medtronic, Minneapolis, USA) were located at the inflow and outflow channels to maintain a unidirectional flow. A cam-type blood pump was developed to drive the blood chamber as shown in Fig. 2(a). The blood pump was $18.8 \mathrm{~mm}$ thick and $86 \mathrm{~mm}$ in diameter. The pump piston was connected to the bottom of the blood chamber. The motion of the piston was determined by a motor driven cam. The displacement, velocity and acceleration of the cam for each 


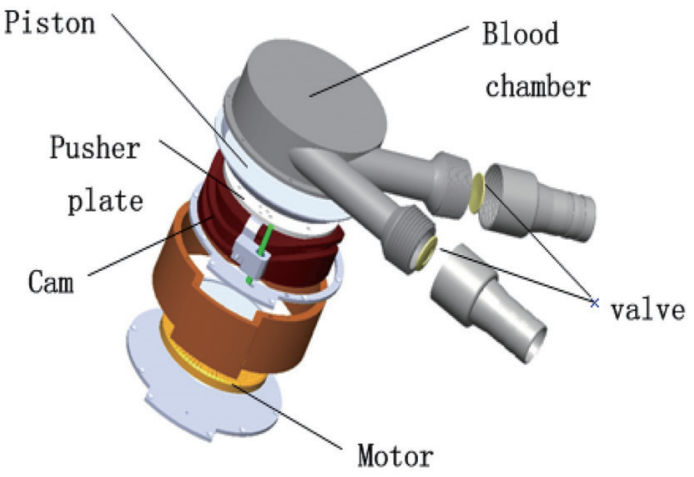

(a)

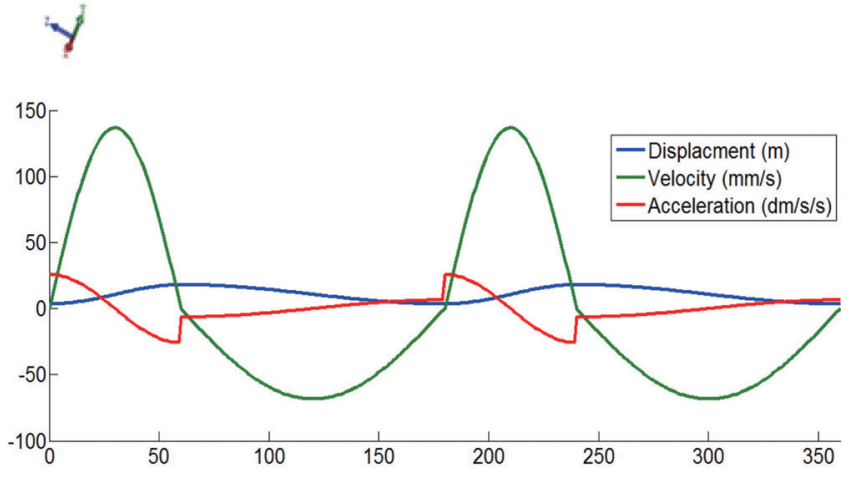

(b)

Fig. 2. Blood pump structure and cam displacement, velocity and acceleration.

revolution were displayed in Fig. 2(b). The velocity would be applied to the moving boundary of the CFD model in the following section.

\subsection{Computational simulation model}

The chamber model was divided into approximately 310000, 530000, 820000 tetrahedral elements with packed densely near the walls by the integrated computer engineering and manufacturing code for computational fluid dynamics (ICEMCFD) to verify the independence of grid. The calculation showed no obvious difference with the variation of grid quantity. The cut plane of the mesh was shown in Fig. 3 . The CFD analysis was conducted by Fluent with the RNG $k-\varepsilon$ turbulent model [17] in the ANSYS 14.0 (ANSYS Inc. Pittsburgh, Pennsylvania) workbench. A fluid density of $1.063 \mathrm{~g} / \mathrm{mm}^{3}$ and a viscosity of $3.5 \times 10^{-6} \mathrm{~m}^{2} / \mathrm{s}$ were used for the blood simulation. Pathlines were used to visualize the flow state in blood chamber. A dynamic mesh method was used to model the piston motion. The motion profile was in accordance with the cam profile shown in Fig. 2. The beat rate was set as 75 beats per minute, which was consistent with the normal heart rate. The filling phase lasted $0.5 \mathrm{~s}$ and ejection phase lasted $0.3 \mathrm{~s}$. During the filling phase, a preload of $10 \mathrm{mmHg}$ was applied to the inflow channel while the outflow channel was occluded. During the ejection phase, an afterload of $100 \mathrm{mmHg}$ was applied to the outflow channel while the inflow channel was occluded. In post-processing, velocity, wall shear stresses and scalar wall strain rates were investigated. Scalar wall strain rates values were defined according to Eq. (1) [18].

$$
\gamma=\frac{1}{\sqrt{3}} \sqrt{\varepsilon_{x x}^{2}+\varepsilon_{y y}^{2}+\varepsilon_{z z}^{2}-\varepsilon_{x x} \varepsilon_{y y}-\varepsilon_{y y} \varepsilon_{z z}-\varepsilon_{x x} \varepsilon_{z z}+3\left(\varepsilon_{x y}^{2}+\varepsilon_{y z}^{2}+\varepsilon_{x z}^{2}\right)}
$$

Where $\varepsilon_{i j}=\frac{1}{2}\left(\frac{\partial \mu_{i}}{\partial \chi_{j}}+\frac{\partial \mu_{j}}{\partial \chi_{i}}\right), \chi_{i}$ and $\mu_{i}$ are the cartesian coordinate and velocity component in the $i^{\text {th }}$ direction, respectively.

\subsection{In vitro PIV validation model}

Experimental validation was conducted using Dynamic Studio compact TR-PIV system (Dantec, Copenhagen, Denmark), which combines the Ray Power lasers with Dantec Dynamics' sensitive time resolved imaging Speed Sense cameras. User control and data manipulation was executed through Dynamic Studio. The pump was connected to a mock circulatory loop (first described by Rosenberg et 


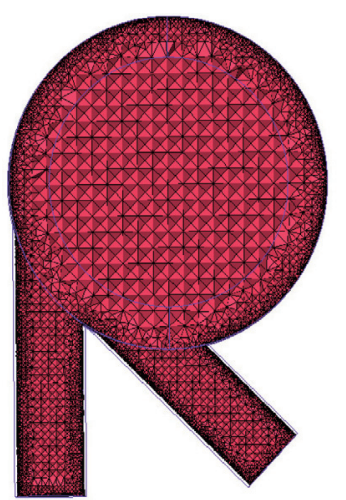

Fig. 3. Cut plane of the fluid domain with a densified near wall grid.

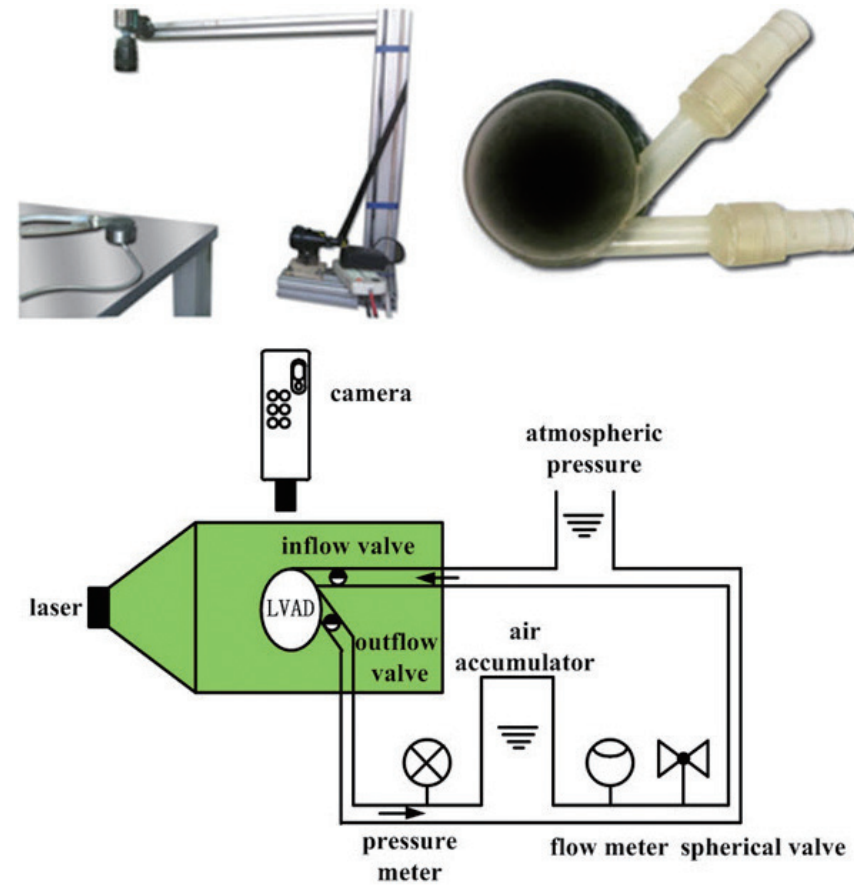

Fig. 4. Schematic diagram of the experimental setup used to conduct the PIV studies.

al. [19]) at a working condition of 5 liters per minute against $100 \mathrm{mmHg}$ afterload. A mixture of water $(62.5 \%)$ and glycerin $(37.5 \%)$ was used to simulate the Newtonian kinematic blood viscosity at $22^{\circ} \mathrm{C}$. The refractive index of the fluid $(n=1.43)$ was close to that of the blood chamber (Polyurethane, $n=1.45$ ). Reflective silver coated hollow vitreous spheres (Conduct, Potters Industries Inc., Carls USA) with a diameter of $200 \mu \mathrm{m}$ were added to the fluid for flow visualization. A quantum laser at $0.65 \mathrm{~W}$ was used to generate the laser sheet. A high speed camera with a frame rate of $2080 \mathrm{fps}$ and a 1280 pixels $\times 1024$ pixels resolution was used to record the flow patterns. The resulting images were post-processed in Dynamic Studio to quantify the velocity counters averaged over 50 cycles using adaptive cross-correlation algorithm. A schematic diagram of the experimental setup used to conduct the PIV studies was shown in Fig. 4.

\section{Results}

\subsection{Computational fluid dynamics and echocardiography comparison}

Figures 5(a) and (b) showed pathlines during the filling and ejection phases of the CFD model. Pathlines used to visualize the flow in the chamber clearly showed a 3-dimensional vortex. The rotational vortex maintained a counter-clockwise rotation throughout the pulsatile period. The fluid swirled along the pump structure fluently. Figure 6 (row1, row2) showed in plane velocity contours and velocity vectors $3 \mathrm{~mm}$ from the front face in a pump period. There was very small area with low velocity shifting around the geometrical center of blood pump due to the vortex. During the initial filling phase, a jet flow appeared at the inflow interface $(100 \mathrm{~ms})$. As the filling phase progressed, the jet strength grew and 


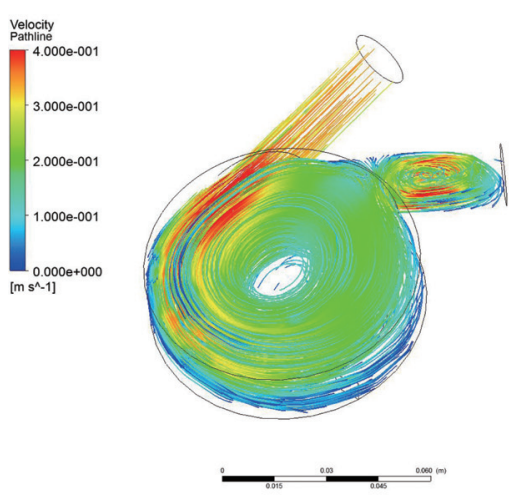

(a)

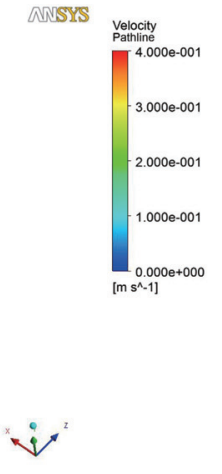

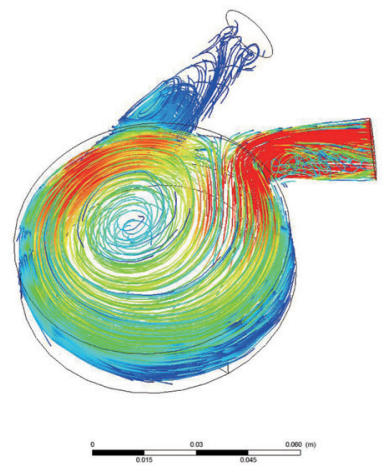

(b)

Fig. 5. Particle pathlines during filling phase (a) and ejecting phase (b).

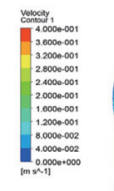

$00 \mathrm{~ms}$
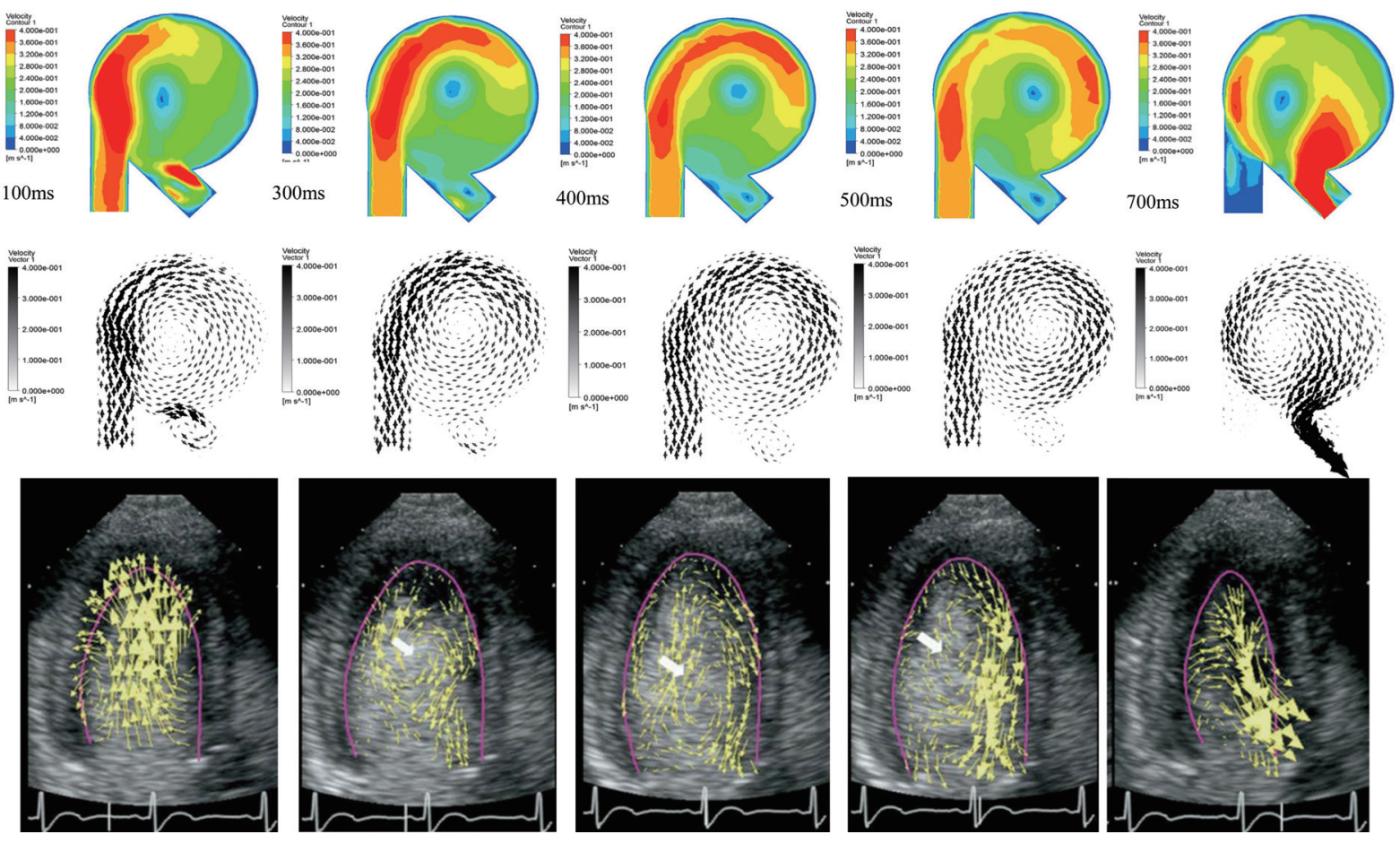

Fig. 6. Computational velocity counters (row1), velocity vectors (row2) and echocardiography in human left ventricular using vector particle image velocimetry (row3) during a pulsatile period.

vortex began to form $(300 \mathrm{~ms})$. A large fully developed vortex formed through the middle to the end of the filling phase (400 ms, $500 \mathrm{~ms}$ ). During the ejection phase, the fluid with the maximum velocity was directed by the arrangement of the outflow channel and ejected out $(700 \mathrm{~ms})$. The vortex formed during the filling phase sustained, resulting a continuous and smooth transition between filling and ejection phase. Therefore the kinetic energy of the vortex was fully utilized to facilitate the blood ejection until the next filling phase. Using echocardiographic particle image velocimetry, Fig. 6 (row3) showed the echocardiography in normal human left ventricle during a pulsatile period studied by Geu-Ru Hong et 


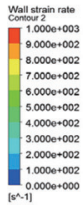

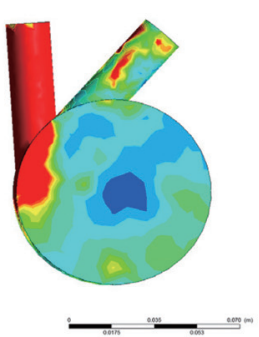

(a)
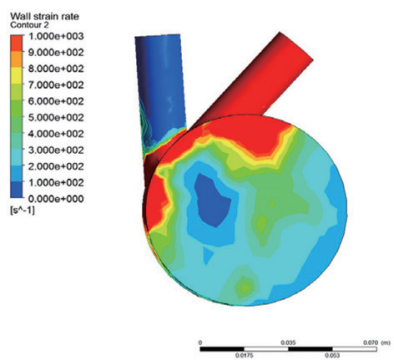

(b)

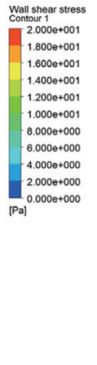

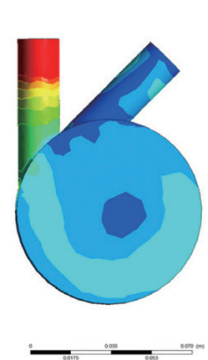

(c)

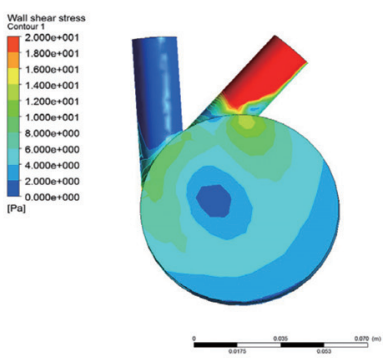

(d)

Fig. 7. Scalar wall strain rates (a, b) and wall shear stresses (c, d) in the middle of filling phase (400 ms) and ejecting phase (700 ms).

al. (permission granted by Elsevier) [20]. Comparison between echocardiography in human left ventricle and CFD results were shown in Fig. 6. It was found that the fluid in the asymmetric blood chamber formed a global vortex flow. The characteristics of initial jet flow, form and development of the vortex and utilizing the redirected flow to facilitate the kinetic energy of the vortex were much similar to those in the human left ventricle.

Thrombus deposition decreased on polyurethane when the wall-strain rates rise above $500 \mathrm{~s}^{-1}$ [21]. Usually the inflow, outflow and the connection part of the chamber of artificial LVADs were zones more prone to produce thrombosis deposition due to low wall-strain rates. Contours of scalar wall strain rates calculated by Eq. (1) in the middle of filling and ejection phase were shown in Figs 7(a) and (b). CFD results showed that wall strain rates higher than $500 \mathrm{~s}^{-1}$ mainly concentrated on the inflow, outflow and the connection part of the vortex bionic chamber. That was coincident with the thrombus high incidence areas mentioned above. These characteristics were in favor of decreasing the thrombus deposition objectively.

The contours of wall shear stresses in the middle of filling phase and ejection phase are presented in Figs 7(c) and (d). The region with maximum wall shear stresses magnitudes corresponded to the region of maximum velocity contours. Higher wall shear stresses zones mainly concentrated on the inflow and outflow channel. Benefiting from the regularity of the vortex flow, wall shear stresses in most part of the blood chamber was lower than $16 \mathrm{~Pa}$. And the minimum wall shear stresses located at the center of the vortex.

\subsection{PIV results and validation}

The PIV results were processed in the DynamicStudio. Figure 8 row 1 and row 2 showed the in-plane velocity magnitude contours $3 \mathrm{~mm}$ from the front face for both the PIV measurements and computations from early diastole to systole. Both displayed similar inlet channel jets at the beginning of the filling phase. And the vortex formed in the filling process was redirected to the outlet channel during the ejection phase. The rotational flow patterns and shifting vortex center were also similar in the whole blood chamber. The vortex strength was quantified by the mean velocity in an annular area covering $25 \mathrm{~mm}-35 \mathrm{~mm}$ of the chamber radius as shown in Fig. 9(a). The computational and experimental vortex velocity in a pulsatile period was shown in Fig. 9(b). The computational results matched well with experimental results. From Fig. 9(b), it was found that the vortex was getting stronger as the filling phase proceeded. The vorticity of the moving vortex in the chamber decreased slowly during pump ejection. 


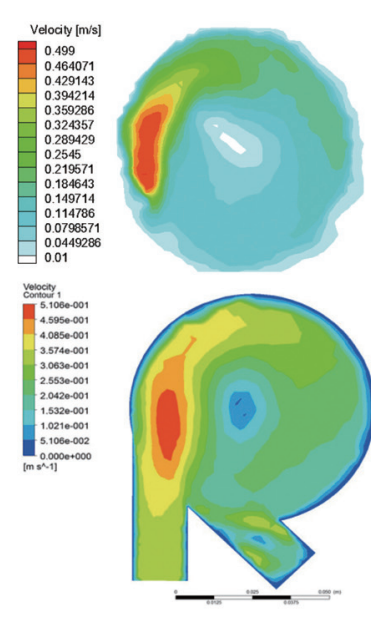

$200 \mathrm{~ms}$
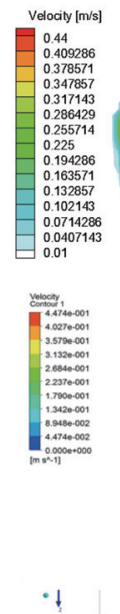

(1)
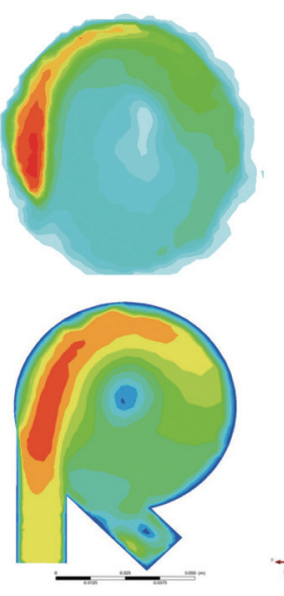

$300 \mathrm{~ms}$
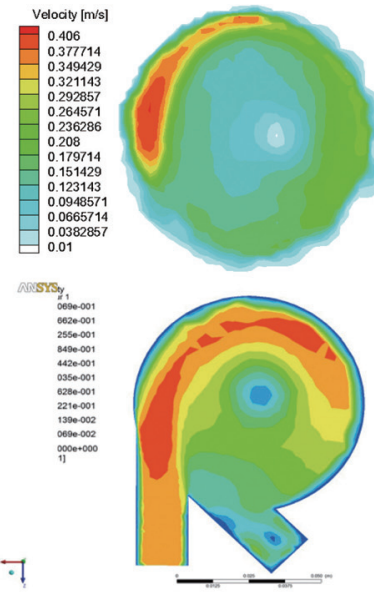

$400 \mathrm{~ms}$
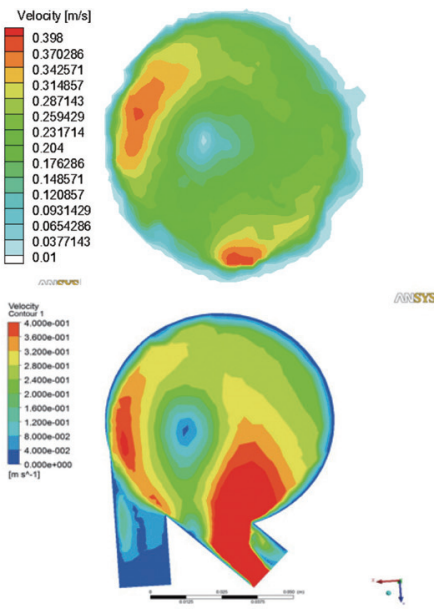

$700 \mathrm{~ms}$

Fig. 8. Velocity counters of experimental (row 1) and computational (row 2) results comparison.

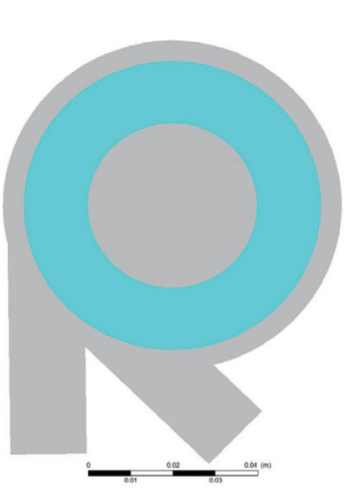

(a)

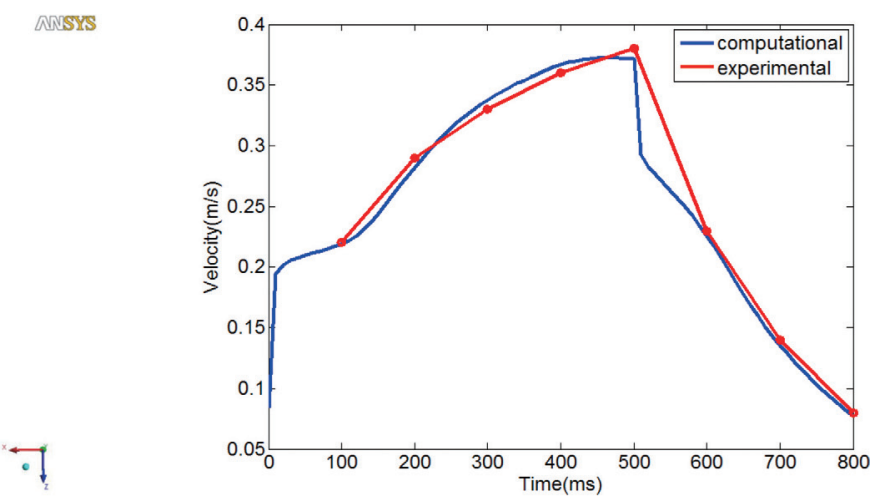

(b)

Fig. 9. Vortex strength in a pulsatile period.

\section{Discussion}

\subsection{Computational fluid dynamics}

The ideal flow state in LVAD should simulate the flow pattern in the human left ventricle, which was natural optimized. During the diastolic phase, the flow was primarily characterized by a strong compact jet that entered the ventricle through the mitral orifice and formed a vortex pattern. In systole, the rotational flow was redirected to the aorta to form a persistent recirculating flow state. This recirculating pattern in left ventricle was considered to act as a sort of reservoir that stored some kinetic energy and facilitated blood ejection [22-24]. From the CFD results, it was found that channels arranged in asymmetric fashion with a $45^{\circ}$ intersection angle on blood chamber can closely reproduce the initial jet flow, development of the vortex and the redirected flow to facilitate the kinetic energy of the vortex similar to a native ventricle. The vortex was fully developed in the global blood chamber. Transition between filling and ejection phase was fluent and stable. The vortex kept in a consistent rotational direction due to the 
bionic cardiovascular structure of the flow channels arrangement avoiding the interruption and dissipation of the vortex flow kinetic energy. This vortex flow pattern was much similar to those in the human left ventricle which provided a good washing effect to prevent thrombus and facilitate blood ejection.

The piston motion was implemented by applying a dynamic mesh to emulate the chamber volume variation. Comparing with PIV results, the dynamic mesh model was found to accurately reproduce the processes of the piston motions. A discrete method was used to model the valve closure, where the valves were fixed as fully opened or occluded through the simulation. We assumed that the dynamic valve closure effects were of secondary importance to the flow within the chamber. The reason was that the transition of opening and closing was short compared to the duration of systole and diastole [25|26]. Newtonian blood fluid was used in the CFD model which was consistent with CFD studies of current mechanical circulatory support devices including VAD in the literature [27].

\subsection{Particle image velocimetry}

The PIV experiment was conducted to verify the continuous vortex flow predicted by the CFD. Both qualitative comparison of the flow pattern and quantitative velocity histories in a pulsatile period demonstrated a high level of agreement between the computational and experimental results. The center of the vortex was an area of low swirl speed, which might be a potential area for blood stagnation. However, the center of the vortex shifted constantly during the pulsatile period, avoiding blood stagnation at a single location that could minimize the risk of thrombosis in the pump. The main observed differences were the fluctuations present in the instantaneous CFD results, which were absent from the averaged PIV results. However, the overall CFD flow patterns closely resembled the in vitro chamber flow. Based on the quality of the validation data, it was possible to extend the analysis to CFD results where experimental data were not available such as the 3-dimentional vortex pathlines in the blood chamber. The PIV results were 2-dimensional images. In the future, it would be desirable for a volumetric PIV experiment for 3-dimentional comparison.

\section{Conclusion}

Using CFD models, this work explored an asymmetric channels arrangement with a $45^{\circ}$ intersection angle between inflow and outflow channels imitating the vascular structure on left ventricle. A model with these characteristics would generate continuous vortex flow to approximately imitate the intraventricular flow. This vortex flow pattern had a good washing effect and could be promising for the development of pulsatile LVAD. The model was validated against in vitro PIV measurements. The quantitative flow velocity and the qualitative flow pattern matched well between the computational and experimental results.

\section{Acknowledgment}

Project was supported by National Natural Science Foundation of China (Grant No. 81027001, 51275287). 


\section{References}

[1] Valika AA, William C. A review of long-term mechanical circulatory support as destination therapy: Evolving paradigms for treatment of advanced heat failure. ISRN Transplantation. 2012; 2013: 1-5.

[2] Kirklin JK, Naftel DC, Kormos RL, Stevenson LW, Pagani FD, Miller MA et al. The fourth INTERMACS annual report: 4,000 implants and counting. J Heart Lung Transplant. 2012; 31: 17-126.

[3] Huang H, Yang M, Lu C, Xu L, Zhuang X, Meng F. A numerical method to enhance the performance of a cam-type electric motor-driven left ventricular assist device. Artif Organs. 2013; 37: 875-883.

[4] Fang JC. Rise of the machines-left ventricular assist devices as permanent therapy for advanced heart failure. N Engl J Med. 2009; 361: 2282-85.

[5] Krabatsch T, Schweiger M, Dandel M, Stepanenko A, Drews T, Potapov E et al. Is bridge to recovery more likely with pulsatile left ventricular assist devices than with nonpulsatile flow systems. Ann Thorac Surg. 2011; 91: 1335-1340.

[6] Crow S, John R, Boyle A, Shumway S, Liao K, Adams MC et al. Gastrointestinal bleeding rates in recipients of nonpulsatile and pulsatile left ventricular assist devices. J Thorac Cardiovasc Surg. 2009; 137: 208-215.

[7] Ji BY, Undar A. An evaluation of the benefits of pulsatile versus nonpulsatile perfusion during cardiopulmonary bypass procedures in pediatric and adult cardiac patients. ASAIO J. 2006; 52: 357-61.

[8] Champsaur G, Parisot P, Martinot S, Ninet J, Robin J, Ovize M et al. Pulsatility improves hemodynamics during fetal bypass:Experimental comparative study of pulsatile versus steady flow. Circulation. 1994; 90: 1147-50.

[9] Bartoli CR, Giridharan GA, Litwak KN et al. Hemodynamic responses to continuous versus pulsatile mechanical unloading of the failing left ventrical. ASAIO J. 2010; 56: 410-416.

[10] Arvand A, Hormes M, Reul H. A validated computational fluid dynamics model to estimate hemolysis in a rotary blood pump. Artif Organs. 2005; 29: 531-40.

[11] Mitamura Y, Nakamura H, Sekine K, Kim DW, Youzo R et al. Prediction of hemolysis in rotary blood pumps with computational fluid dynamics analysis. J Congest Heart Fail Circ Support. 2001; 1: 331-336.

[12] Demir O, Biyikli E, Lazoglu I, Kucukaksu S. Design of a centrifugal blood pump: Heart turcica centrifugal. Artif Organs. 2011; 35: 7.

[13] Okamoto E, Hashimoto T, Mitamura Y. Design of a miniature implantable left ventricular assist device using CAD/CAM technology. J Artif Organs. 2003; 6: 162-167.

[14] Kreider JW, Manning KB, Oley LA, Fontaine AA Steven D. A parametric study of valve orientation flow dynamics. ASAIO J. 2006; 52: 123-131.

[15] Giridharana GA, Ledererb C, Berthec A, Goubergrits L, Hutzenlaub J, slaughter MS et al. Flow dynamics of a novel counter pulsation device characterized by CFD and PIV modeling. Med Eng Phy. 2011; 33: 1193-1202.

[16] Gianni P, Federico D, Nature optimizes the swirling flow in the human left ventricle. Phys Rev Lett. 2005; 95: 108101108104.

[17] Esmaiil G, Entropy generation in transitional boundary layers, LAP LAMBERT Academic Publishing, 2013.

[18] Medvitz RB, Kreider JW, Manning KB, Fontaine AA, Steven D, Paterson EG. Development and validation of a computational fluid dynamics methodology for simulation of pulsatile left ventricular assist devices. ASAIO J. 2007; 53: $122-131$.

[19] Medvitz RB, Reddy V, Deutsch S, Manning KB, Paterson EG. Validation of a CFD methodology for positive displacement LVAD analysis using PIV data. J Biomech Eng. 2009; 131: 1110091-1110098.

[20] Reprinted from characterization and quantification of vortex flow in the human left ventricle by contrast echocardiography using vector particle image velocimetry, Vol.6, Geu-Ru Hong, Gianni Pedrizzetti, Giovanni Tonti, Peng Li, Zhao Wei, Jin Kyung Kim et al. 705-717, Copyright (2008), with permission from Elsevier.

[21] Hubbell JA, McIntire LV. Visualization and analysis of mural thrombogenesis on collagen, polyurethane, and nylon. Biomaterials. 1986; 7: 354-363.

[22] Gianni P, Federico D, Giovanni T. On the left ventricular vortex reversal after mitral valve replacement. Annals of Biomedical Engineering. 2010; 38: 769-773.

[23] Philip JK, Yang G-Z, Wilkes AJ, Mohiaddin RH, Firmin DN, Yacoub MH. Asymmetric redirection of flow through the heart. Nature. 404: 759-761.

[24] Giorgiobolzon, Luiginozovatto, Gianni P. Birth of three-dimensionality in a pulsed jet through a circular orifice. J. Fluid Mech. 2003; 493: 209-218.

[25] Stijnen JMA, Hart JD, Bovendeerd PHM, van de Vosse FN. Evaluation of a fictitious domain method for predicting dynamic response of mechanical heart valves. J Fluids Struct. 2004; 19: 835-850.

[26] Stijnen J. Interaction between the mitral and aortic heart valve: An experimental and computational study [dissertation]. Eindhoven: Eindhoven University; 2004.

[27] Behbahani M, Behr M, Hormes M et al. A review of computational fluid dynamics analysis of blood pumps. Eur J Appl. 2009; 20: 363-397. 